\title{
EFEITO DO NÚMERO DE JOGADORES NAS AÇÕES TÉCNICO-TÁTICAS EM JOGOS REDUZIDOS NO FUTEBOL
}

\author{
Marcelo Figueiró Baldi ${ }^{1}$ \\ Juliana Roberta da Silva ${ }^{2}$ \\ Bruna Scotti Faria ${ }^{2}$
}

BALDI, M. F.; SILVA, J. R. da; FARIA, B. S. Efeito do número de jogadores nas ações técnico-táticas em jogos reduzidos no futebol. Arq. Cienc. Saúde UNIPAR, Umuarama, v. 21, n. 3, p. 169-174, set./dez. 2017.

\begin{abstract}
RESUMO: Os jogos reduzidos constituem a base de muitos programas de formação no futebol. A partir destes jogos, são realizadas modificações com o intuito de estimular um determinado fluxo de ações (técnico-táticas) em contexto real de jogo. Entre as principais estratégias está o aumento ou diminuição do número de jogadores para um determinado tamanho de campo. Contudo, ainda hoje, apesar da ampla utilização de modificações de jogos reduzidos como estratégia de ensino, aprendizagem e treinamento no futebol estas intervenções carecem de demonstração científica acerca da sua utilidade e eficácia. Esta falta de evidências empíricas perpetua o famigerado abismo entre teoria e prática e a construção de atividades de treino, unicamente, baseada na subjetividade do treinador. Assim, o objetivo do presente estudo foi analisar o efeito do número de jogadores na frequência e distribuição das ações técnico-táticas em uma escolinha de futebol e futsal. Participaram do estudo 18 jogadores de uma escolinha de futebol e futsal do Paraná. As situações analisadas foram 4vs4 + goleiros e 5vs5 + goleiros. A análise das ações técnico-táticas foi adaptada dos critérios originalmente propostos no Team Sport Assessment Procedure (TSAP). Os jogos foram gravados em câmera digital e posteriormente analisados em vídeo. De modo geral, os resultados demostraram maior frequência de ações técnico-táticas em situação de 4vs4 + goleiros comparada a situação de 5vs5 + goleiros. Contudo é necessário a intervenção do treinador, incluindo modificações destes jogos reduzidos, para permitir exercitação mais homogênea entre os jogadores.

PALAVRAS-CHAVE: Desenvolvimento técnico-tático. Futebol. Jogos reduzidos.
\end{abstract}

\section{EFFECT OF THE NUMBER OF PLAYERS IN TECHNICAL-TACTICAL ACTIONS IN REDUCED FOOTBALL GAMES}

ABSTRACT: Small-sided games form the basis of many youth soccer programs. From these games, modifications are made in order to stimulate a certain flow of technical-tactical actions in real game context. Among the most common strategies are the increase or decrease of the number of player to a given field size. However, despite the wide use of small-sided games as a teaching strategy in soccer, these interventions lack of scientific evidence to demonstrate their utility and efficacy. This lack of evidence strengthens the gap between theory and practice and the design of small-sided games based, solely, on coaches' subjectivity. Thus, the present study analyzed the effect of the number of players on the frequency and distribution of technical-tactical actions in a soccer academy. Participated in the study 18 players from a soccer and futsal academy from Paraná. Situations analyzed were 4vs4 + goalkeepers and 5vs5 + goalkeepers. Analysis of technicaltactical actions were adapted from the criteria originally proposed in the Team Sport Assessment Procedure (TSAP). Games were recorded using a digital camera and saved for posterior computer analysis. Overall, results elicited more frequency of technical-tactical actions in $4 \mathrm{vs} 4+$ goalkeepers compared to 5vs5 + goalkeepers. However, it is needed coach's intervention, including other game modifications, to allow a more homogeneous participation among players.

KEYWORDS: Technical-tactical development. Soccer. Small-sided games.

\section{Introdução}

Jogos reduzidos são adaptações do jogo formal (11 vs 11) (HILL-HASS et al., 2011) amplamente utilizados no processo de ensino, aprendizagem e treinamento nas escolinhas de futebol (BALDI et al., 2016). Isto porque esses jogos ao incluírem, em geral, regras mais simples, menos jogadores e espaço reduzido são inerentes a própria limitação técnico-tática das crianças. Segundo por que a partir destas modificações o professor/treinador pode criar diferentes cenários de prática (GARGANTA, 2006) que estimulam um determinado fluxo de ações (técnico-táticas) em contexto real de jogo (DAVIDS et al., 2013).

Contudo, apesar da coerência e da riqueza de proposições teóricas relativamente a utilização de jogos reduzidos no futebol (ZISKIND, 2011) são escassas as evidências científicas que fundamentam a prática do professor/treinador (FRADUA et al., 2012).

Além disso, o ufanismo propalado em pró da utilização de jogos reduzidos, de modo geral, carece de uma aná- lise científica acerca dos seus pontos negativos e limitações. Neste sentido, na prática do desenvolvimento técnico-tático de crianças, a partir de jogos reduzidos, treinadores mais atentos à Pedagogia do Esporte e críticos (do ponto de vista metodológico) tem duas preocupações centrais. A primeira é de garantir uma alta frequência de exercitação pelo grupo. A segunda é que todos exercitem em igual medida (BALDI et al., 2016).

Dentre as modificações mais comuns para induzir alterações no comportamento técnico-tático do jogador está o número de jogadores (DELLAL et al., 2012). Contudo, como já mencionado, são raros os estudos que tem de fato demonstrado como que a inclusão de jogadores a mais no campo de jogo (para um mesmo tamanho de campo) altera a frequência e distribuição de exercitação das variáveis técnico-táticas (BALDI et al., 2016). A falta de dados a este respeito perpetua, por um lado, o famigerado abismo entre teoria e prática, deixando os treinadores em um "limbo" sem a evidência científica de que determinadas atividades de treino estão, de fato, atingindo os objetivos almejados. Por outro

DOI: 10.25110 /arqsaude.v21i3.2017.5694

${ }^{1}$ Professor Mestre

${ }^{2}$ Professora

Endereço para correspondência: rua Ângelo Colautto, 3020, edifício Apolo I, apartamento 301, bairro Jardim dos Príncipes. Umuarama - PR. CEP 87502046. e-mail: marcelobaldi@unipar.br 
lado, permanece a construção de atividades de treino unicamente baseada na análise subjetiva do professor/treinador.

Diante do exposto, o presente estudo tem como objetivo analisar o efeito do número de jogadores na frequência e distribuição das ações técnico-táticas em uma escolinha de futebol e futsal.

\section{Métodos}

\section{Caracterização do estudo}

Esta pesquisa caracteriza-se como um estudo de caso, quase experimental e transversal (THOMAS; NELSON; SILVERMAN, 2012).

\section{Participantes}

Participaram do estudo 18 jogadores, do sexo masculino, com idade entre 8 e 11 anos de uma escolinha de futebol e futsal do Paraná. Os participantes foram selecionados de forma intencional não probabilística. A filosofia de formação da escolinha de futebol e futsal preconiza a experiência no treinamento alternado nestas duas modalidades. Neste sentido, as crianças treinam, em média, 2 vezes por semana ( \pm 1 hora por sessão). Devido à especificidade da sua função ser distinta dos objetivos do estudo, os goleiros foram excluídos das análises.

\section{Procedimentos para a coleta de dados}

Após obter o parecer favorável do Comitê de Ética em Pesquisa com Seres Humanos (CAEE 31255914.6.0000.0109), foram apresentadas a proposta do estudo, esclarecidos os objetivos e métodos empregados aos participantes e pais ou responsáveis. Na sequência, os termos de consentimento livre e esclarecido foram obtidos dos pais ou responsáveis.

\section{Coleta de dados}

Todas as avaliações foram realizadas no mesmo dia, no período da tarde, em gramado natural e em condições climáticas semelhantes.

\section{Protocolos de avaliação}

Avaliação das ações técnico-táticas em jogos reduzidos

Os indivíduos participaram de 2 jogos reduzidos com configurações diferentes: 4vs4 + goleiros ("quatro contra quatro mais goleiros") e 5vs5 + goleiros ("cinco contra cinco mais goleiros"). Essas configurações de jogos reduzidos foram escolhidas com o intuito de comparar duas possibilidades (de jogos reduzidos) para o desenvolvimento técnico no futebol. Além disso, essa escolha foi baseado na ideia de bom jogo para um determinado nível (GRAÇA, 1996). Em outras palavras, o que se fez foi comparar um jogo reduzido (4vs4 + goleiros) em que os jogadores exibem um nível de organização tática elementar no campo de jogo com outro jogo reduzido (5vs5 + goleiros) que representasse um desafio ao nível técnico-tático dos participantes.

Em todos os jogos, foram mantidas as mesmas dimensões do campo ( $36 \times 27 \mathrm{~m})$, tamanho das balizas $(3 \times 2 \mathrm{~m}) \mathrm{e}$ duração de jogo ( 8 minutos contínuos). Durante todo tempo, 8 acadêmicos (estagiários da escolinha de futebol e futsal) permaneceram ao redor do campo para auxiliar na reposição imediata da bola, caso necessário.
Foi solicitado aos participantes jogar de acordo com as regras oficiais do futebol, com exceção da regra de impedimento. Além disso, para tornar o jogo ainda mais dinâmico, com o mínimo de interrupções, foram suprimidas as cobranças de escanteio e instituída a reposição das cobranças de lateral com os pés. Em adição, a equipe ao sofrer um gol reiniciava as suas ações ofensivas automaticamente com a reposição do goleiro. Todos os jogadores tinham experiência com as configurações de jogo bem como as regras estipuladas.

As equipes foram equilibradas de forma competitiva mediante a avaliação subjetiva dos treinadores, conforme realizam, normalmente, em uma sessão de treinamento. Durante todo o tempo, os treinadores permaneceram posicionados adjacentes ao campo de jogo, supervisionando a atividade diretamente, podendo fornecer estimulo volitivo aos participantes. Todavia, foi solicitado não interromper a atividade ou fornecer instruções táticas ou técnicas.

\section{Instrumentos de medida}

Obtenção e armazenamento das imagens

As imagens foram obtidas com uma câmera de vídeo e armazenadas em computador para posterior análise. A câmera de vídeo foi posicionada no vértice do escanteio, a aproximadamente $10 \mathrm{~m}$ do campo e $5 \mathrm{~m}$ de elevação. Esta posição da câmera foi escolhida por proporcionar o melhor ângulo de visualização do campo e dos jogadores durante os jogos.

\section{Procedimento de Avaliação de Jogos Coletivos}

A avaliação da frequência de ações técnico-táticas durantes as atividades de treino foi adaptada dos critérios estabelecidos no Procedimento de Avaliação de Jogos Coletivos - Team Sport Assessment Procedure (TSAP) (GRÉHAIGNE; GODBOUT; BOUTHIER, 1997). As variáveis analisadas e critérios de avaliações adotados foram:

\section{Bolas recebidas $(\mathrm{BR})$}

Critério: o jogador recebe uma bola de uma companheiro e não perde, imediatamente, o controle dela.

Bolas conquistadas (BC)

Critério: qualquer bola que o jogador obtém sem que seja proveniente de um passe de um companheiro de equipe.

Bolas neutras (BN)

Critério: qualquer passe que não coloca a outra equipe em risco de gol.

Bolas ofensivas (BO)

Critério: passes que colocam a outra equipe em risco de gol.

Finalização (FN)

Critério: são todas as tentativas de finalização ao gol.

\section{Análise dos dados}

Dez observadores treinados com o sistema de avaliação analisaram, classificaram e registraram as ações técni- 
co-táticas em todos os jogos. Cada jogador foi avaliado por um observador em cada jogo de forma aleatória. As situações que geraram dúvida foram discutidas entre os avaliadores até chegar a um consenso. As variáveis foram analisadas em termos de média, amplitude (são os valores extremos, isto é, o maior e menor valor observado entre os jogadores em cada variável) e distribuição dos resultados, ou seja, a frequência de exercitação das ações técnico-táticas pelos jogadores. A análise da distribuição dos resultados foi feita a partir de histogramas. O teste de Pearson foi utilizada para verificar a correlação entre as médias e a amplitude dos resultados.

\section{Resultados}

Na Tabela 1 e nas Figuras de 1 a $\mathbf{6}$ são apresentados os resultados referentes a realização de ações técnico-táticas em jogos reduzidos (4vs4 + goleiros e 5vs5 + goleiros) por crianças de 8 a 11 anos de idade de uma escolinha de futebol e futsal. Especificamente, na Tabela 1 são apresentadas as médias e amplitude dos resultados. Enquanto isso, nas Figuras de 1 a 5 e Figura 6 são ilustradas as distribuições e correlações entre as variáveis, respectivamente.

Tabela 1: Frequência de ações técnico-táticas realizadas em 4vs4 + goleiros e 5vs5 + goleiros por crianças de 8 a 11 anos de idade de uma escolinha de futebol e futsal $(n=18)$.

\begin{tabular}{ccccccccccc}
\hline Variáveis & \multicolumn{2}{c}{ BR } & \multicolumn{2}{c}{ BC } & \multicolumn{2}{c}{ BN } & \multicolumn{2}{c}{ BO } & \multicolumn{2}{c}{ FN } \\
\cline { 2 - 12 } Jogo & $4 v s 4$ & $5 v s 5$ & $4 v s 4$ & $5 v s 5$ & $4 v s 4$ & $5 v s 5$ & $4 v s 4$ & $5 v s 5$ & $4 v s 4$ & $5 v s 5$ \\
\hline Média & 5 & 3,9 & 4,8 & 2,2 & 1,2 & 0,3 & 1,2 & 1,3 & 1,8 & 0,8 \\
Amplitude & $(1-9)$ & $(0-12)$ & $(0-11)$ & $(0-5)$ & $(0-5)$ & $(0-2)$ & $(0-5)$ & $(0-6)$ & $(0-5)$ & $(0-5)$
\end{tabular}

Legenda: $\mathrm{BR}=$ bolas recebidas; $\mathrm{BC}=$ bolas conquistadas; $\mathrm{BN}=$ bolas neutras; $\mathrm{BO}=$ bolas ofensivas; $\mathrm{FN}=$ finalizações. Amplitude $=$ entre parênteses é destacado o menor valor observado (à esquerda) e o maior valor observado (à direita).

De modo geral, é possível observar maior média de ações técnico-táticas em 4vs4 comparado a situação de 5vs5 (Tabela 1). Exceção foi observada apenas na variável Bola Ofensiva cuja média foi superior em $5 \mathrm{vs} 5$.

Foi observada uma grande amplitude na frequência de exercitação das ações técnico-táticas, especialmente nas variáveis Bolas Recebidas e Bolas Conquistadas e uma amplitude moderada em Bolas Ofensivas (5vs5) (Tabela 1 e Figuras 1 a 5) (considerando diferença de 1 à 3 "baixa amplitude"; 4 à 6 "média amplitude" e 7 ou mais "grande amplitude"). Além disso, é importante destacar que em 9 das 10 variáveis analisadas foram registradas frequências nulas de exercitação da ação técnico-tática por pelo menos 1 jogador.

Foram verificadas distribuição não normal em 6 das 10 médias analisadas

Figura 1: Distribuição da frequência das ações de Bolas Recebidas em jogos reduzidos em crianças de 8 a 11 anos de uma escolinha de futebol e futsal.
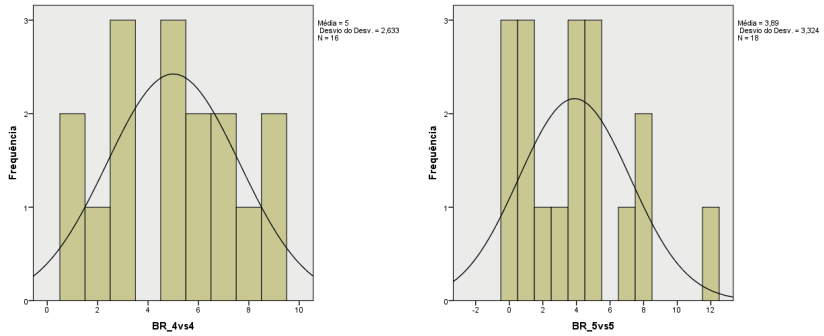

Figura 2. Distribuição da frequência das ações de Bolas Conquistadas em jogos reduzidos em crianças de 8 a 11 anos de uma escolinha de futebol e futsal.
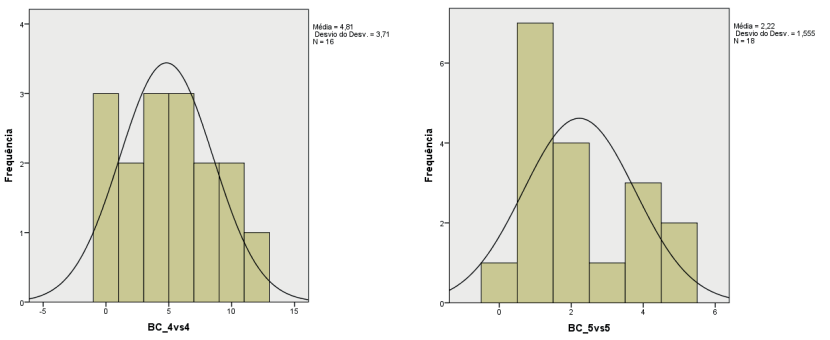

Figura 3. Distribuição da frequência das ações de Bolas Neutras em jogos reduzidos em crianças de 8 a 11 anos de uma escolinha de futebol e futsal.
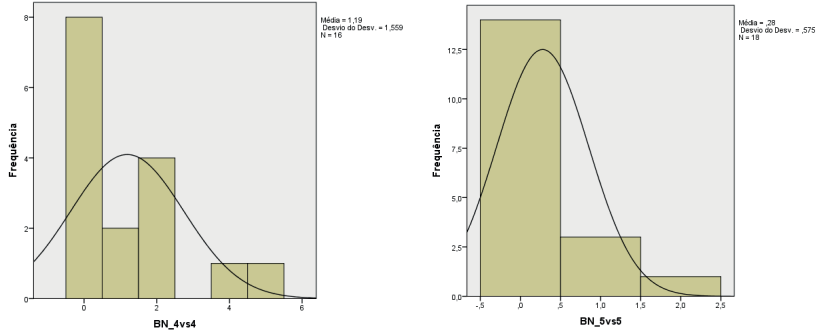

Figura 4. Distribuição da frequência das ações de Bolas Ofensivas em jogos reduzidos em crianças de 8 a 11 anos de uma escolinha de futebol e futsal.

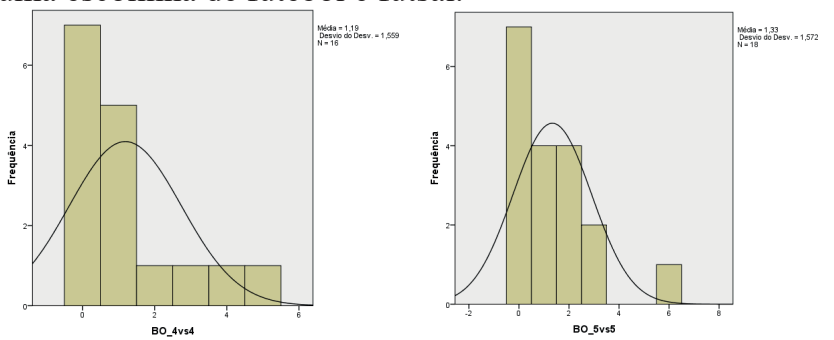


Figura 5. Distribuição da frequência das ações de Finalização em jogos reduzidos em crianças de 8 a 11 anos de uma escolinha de futebol e futsal.
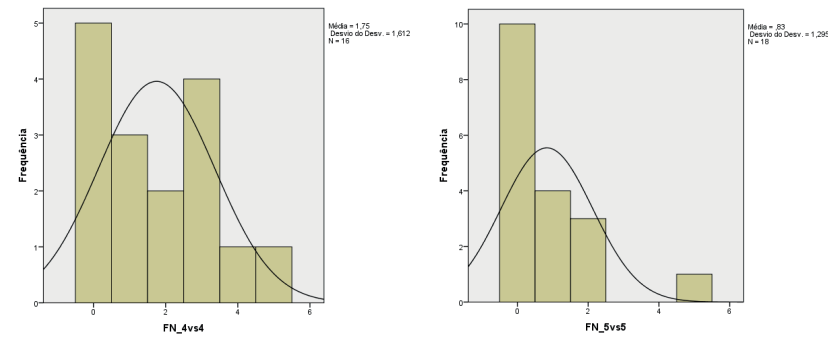

Finalmente, pode-se verificar uma forte correlação $(\mathrm{r}=0,85)$ entre as médias de exercitação das variáveis com a amplitude dos resultados, isto é, quanto maior a média de exercitação de uma determinada ação técnico-tática maior a diferença entre o maior e menor valor observado (Figura 6).

Figura 6: Correlação entre as médias de ações técnico-táticas e a amplitude (diferença entre o maior e menor valor observado) em jogos reduzidos em crianças de 8 a 11 anos de uma escolinha de futebol e futsal $\left(r=0,85 ; R^{2}=0,76\right)$.

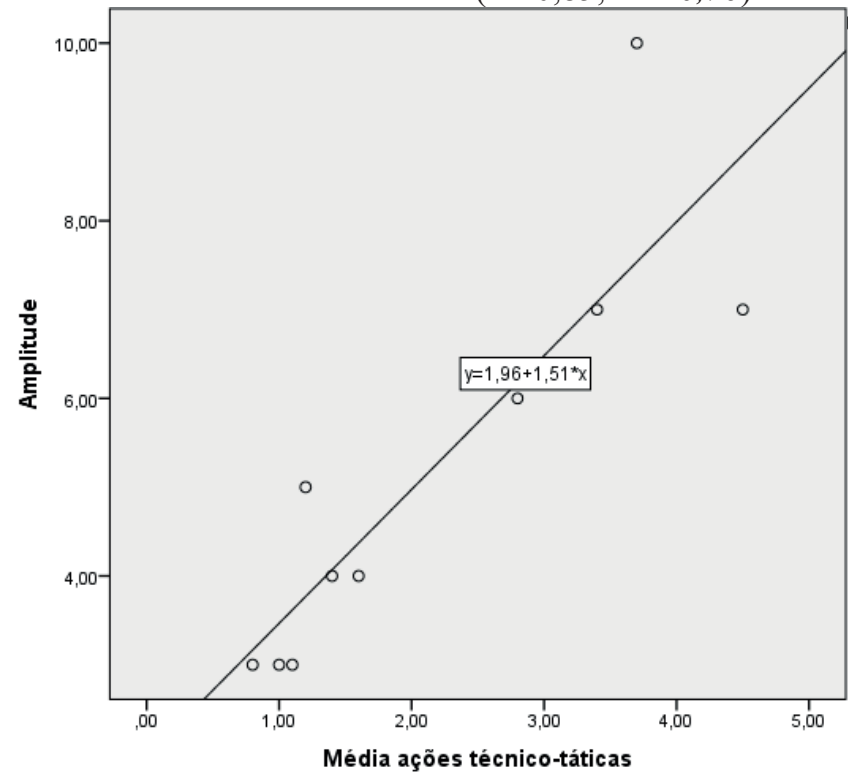

\section{Discussão}

O objetivo do estudo foi analisar o efeito do número de jogadores na frequência e distribuição das ações técnico-táticas em uma escolinha de futebol e futsal. Neste sentido, o principal resultado do estudo foi verificar que a atividade de 4vs4 + goleiros proporciona maior frequência de exercitação das ações técnico-táticas comparada a atividade de 5vs5 + goleiros.

Em relação à análise das ações técnico-táticas em atividades de treino nas categorias de base do futebol, existem inúmeros eventos os quais os observadores podem focar a sua atenção (GRÉHAIGNE; GODBOUT; BOUTHIER, 1997). Contudo, a decisão de quais aspectos devem ser observados, em uma determinada modalidade esportiva, depende da visão dos professores sobre o que os alunos devem aprender (GRÉHAIGNE; GODBOUT; BOUTHIER, 1997). Por trás de cada variável observável de jogo existem informações que importa ao professor ou treinador interpretar
(GRÉHAIGNE; GODBOUT; BOUTHIER, 1997).

De modo geral, foi verificado maior frequência de ações técnico-táticas em 4vs4 comparado a 5vs5. Estas evidências são importantes e confirmam as proposições teóricas e o raciocínio lógico de que, em linhas gerais, jogos com menos jogadores proporcionam mais participação no jogo (GRAÇA, 1998) isto é, maior contato efetivo com a bola.

Resultados análogos a estes foram obtidos em crianças da mesma faixa etária porém, comparando as situações de 3vs3 + goleiros e 4vs4 + goleiros (BALDI et al., 2016). Para o treinador isto significa que, de modo geral, jogos de 4vs4 devem ser utilizados preferivelmente em detrimento as atividades de 5vs5 + goleiros para o desenvolvimento técnico-tático das variáveis analisadas. Além disso, foi observado, a partir dos comportamentos dos jogadores no campo de jogo, que a atividade de 5v5 + goleiros está acima no nível técnico-tático deste grupo. Desta forma, indo contra a ideia de operacionalizar um bom jogo para um determinado nível (GRAÇA, 1998). Sendo possível perceber a desorganização no campo de jogo e a excessiva aglutinação em torno da bola (GARGANTA et al., 2013). Portanto, a atividade de 5vs5 analisada não é compatível com a idade/estágio de desenvolvimento dos praticantes pois o nível de dificuldade e complexidade da atividade está fora no alcance destas crianças (MARTENS, 2004).

$\mathrm{Na}$ análise por variáveis, foi verificado maior frequência de bolas recebidas em situação de 4vs4 comparado a situação de 5vs5 (Tabela 1). Assim como demonstrado por Baldi et al. (2016), ao incluir um jogador a mais no campo de jogo, aumentam as opções de passe ao portador da bola e isso significa menos chances de cada um dos jogadores de receber um passe para a mesma unidade de tempo.

Foi verificado o dobro da frequência de Bolas Conquistadas em 4vs4 comparada a situação de 5vs5. Estes resultados, também são coerentes com a noção de que menos jogadores no campo de jogo proporcionam mais chances de conquistar a bola (BALDI, 2014). Estes resultados apontam em direção diferente do que já havia sido relatado anteriormente na literatura científica. Diferente do observado na presente pesquisa, não havia sido verificado diferença na frequência de Bolas Conquistadas em atividades com número de jogadores diferentes (3vs3 + goleiros comparado a 4vs4 + goleiros) (BALDI et al., 2016). Na comparação com o estudo anterior, pode-se especular que o tempo reduzido de jogo (4 min) impediu maior frequência do fenômeno observado e possivelmente na diferenciação da frequência de exercitação entre as duas situações de jogo reduzido analisadas.

De qualquer maneira, esses resultados revelam a utilidade da atividade de 4vs4 para desenvolver a capacidade defensiva dos jogadores, isto é, a capacidade do jogador contribuir positivamente na possibilidade da equipe recapturar a bola e reiniciar as suas ações de jogo (GRÉHAIGNE; GODBOUT; BOUTHIER, 1997) comparado a situação de 5vs5. Parte destes resultados deve-se ao fato de que, na medida em que o treinador diminui o número de jogadores em campo proporciona aos jogadores se envolverem no centro de jogo e estimula o princípio tático de contenção (TEOLDO et al., 2011).

Na sequência, foi observado uma frequência 4 vezes maior de Bolas Neutras em 4vs4 comparado a situação de 5vs5. Estes resultados são contrários aqueles relatados 
anteriormente na literatura (BALDI et al., 2016). No estudo de Baldi et al. (2016) havia sido foi verificado mais Bolas Neutras em 4vs4 comparada a $3 \mathrm{vs} 3$. Na oportunidade, os pesquisadores perceberam que esta tomada de decisão dos jogadores estava, provavelmente, relacionada à diminuição dos espaços de jogo. Isto é, conforme o aumento do número de jogadores para uma mesma área de jogo, diminuíam as possibilidades de infiltrar na defesa adversária, realizar passes ofensivos (VACARI, 2015) ou finalizar a gol (SOARES, 2015). Com isso, fomentando um jogo de posse de bola (COLLET, 2012) de passes neutros que ofereciam menos riscos a equipe adversária (GRÉHAIGNE; GODBOUT; BOUTHIER, 1997, 1997).

Contudo, os dados da presente pesquisa demonstram o contrário. Neste caso, pode-se especular que dada a exacerbada aglomeração em torno da bola, na situação de $5 \mathrm{vs} 5$ comparada a situação de $4 \mathrm{vs} 4$, os jogadores perceberam a dificuldade em manter linhas de passe. A partir daí optaram por conduzir mais a bola, de modo individualista, para se "livrar" da aglomeração de jogadores em campo de jogo em direção aos espaços livres até perder a posse de bola.

Contrário ao observado em relação as demais variáveis, houve uma mínima diferença na frequência de Bolas Ofensivas em favor da situação de 5vs5. Estes resultados são contrários aos já relatados anteriormente (BALDI et al., 2016). Em relação a esses achados, seria razoável esperar que conforme o aumento do número de jogadores para uma mesma área de jogo, as equipes na defesa passassem a ocupar uma área efetiva de jogo maior (GRÉHAIGNE; CATY; GODBOUT, 2010), tanto em amplitude (de um lado ao outro do campo) como em profundidade (ZISKIND, 2011) e isto dificultaria as oportunidades de passes ofensivos. Além disso, com um jogador a mais em cada uma das equipes seria esperado a diminuição das possibilidades de receber bolas nas costas do último jogador em profundidade (BALDI, 2014). Entretanto, este fato não foi observado. Dessa forma, estes resultados corroboram a noção de que a adaptação dos jogadores a um determinada configuração do jogo é, de fato, um fenômeno complexo e não linear, sujeita aos constrangimentos específicos que agem sobre a equipe e o jogador (DAVIDS et al., 2013).

Foi verificada maior frequência de finalizações em situação de 4vs4 comparada a situação de 5vs5. Neste caso, os resultados corroboram as suposições teóricas e as evidências empíricas de que menos jogadores no campo de jogo proporcionam mais chances de finalização à baliza (SOARES, 2015).

Finalmente, foi observado uma grande amplitude na frequência de exercitação das ações técnico-táticas, especialmente nas variáveis Bolas Recebidas e Bolas Conquistadas (Tabela 1 e Figuras 1 a 5). Assim como uma forte correlação entre as médias de exercitação das variáveis técnico-táticas com a amplitude dos resultados (Figura 6). Além disso, foi verificado distribuição não normal na frequência de exercitação das ações técnico-tática na maioria das variáveis analisadas. Esses resultados demonstram que apesar das vantagens do desenvolvimento técnico-tático em contexto real de jogo cabe ao treinador utilizar modificações das regras para permitir exercitação mais homogênea entre os jogadores.

\section{Conclusão}

Conclui-se que a atividade de 4vs4 + goleiros é mais eficaz que a atividade de 5vs5 + goleiros para o desenvolvimento dos objetivos técnico-táticos, destacados por este estudo, nesta escolinha de futebol. Contudo, é necessário a intervenção do treinador, incluindo modificações destes jogos reduzidos, para permitir exercitação mais homogênea entre os jogadores.

\section{Referências}

BALDI, M. F. Treino Tático no Futebol: efeito das modificações de jogos reduzidos. 2014. 97 f. Dissertação (Mestrado em Educação Física) - Universidade Federal de Santa Catarina, Florianópolis, 2014.

BALDI, M. F. et al. Análise das ações técnico-táticas em jogos reduzidos no futebol. Arquivos de Ciências da Saúde da Unipar, v. 20, n. 1, 2016.

COLLET, C. The possession game? A comparative analysis of ball retention and team success in European and international football, 2007-2010. Journal of Sports Sciences, p. 1-14, 2012.

DAVIDS, K.; ARAÚJO, D.; CORREIA, V.; VILAR, L. How small-sided and conditioned games enhance acquisition of movement and decision-making skills. Exercise and Sport Sciences Review, v. 41, n. 3, 2013.

DELLAL, A. et al. J. Technical and physical demands of small vs. large sided games in relation to playing position in elite soccer. Human Movement Science, n. 31, p. 957-969, 2012.

FRADUA, L. et al. Designing small-sided games for training tactical aspects in soccer: extrapolating pitch sizes from full-size professional matches. Journal of Sports Sciences, 2012.

GARGANTA, J. Ideias e competências para "pilotar" o jogo de futebol. In: TANI, G.; BENTO, J. O.; PETERSEN, R. D. S. Pedagogia do Desporto. Rio de Janeiro: Guanabara Koogan, 2006, p. 313-325.

GARGANTA, J. et al. Fundamentos e práticas para o ensino e treino do futebol. In: TAVARES, F. Jogos Desportivos Coletivos: ensinar a jogar. Porto: FADEUP, 2013, p. 199263.

GRAÇA, A. Os comos e os quandos no ensino dos jogos. In: GRAÇA, A.; OLIVEIRA, J. O ensino dos jogos desportivos. Porto: Rainho \& Neves, 1998, p. 27-34.

GRÉHAIGNE, J. F.; CATY, D.; GODBOUT, P. Modelling ball circulation in invasion team sports: a way to promote learning games through understanding. Physical Education and Sport Pedagogy, v. 15, n. 3, p. 257-270, 2010.

GRÉHAIGNE, J. F.; GODBOUT, P.; BOUTHIER, D. Performance assessment in team sports. Journal of 
Teaching in Physical Education, n. 16, p. 500-516, 1997.

HILL-HASS, S. et al. Physiology of small-sided games training in football: a systematic review. Sports Medicine, v. 41, n. 3, p. 199-220, 2011.

MARTENS, R. Successful coaching. 3. ed. Estados Unidos da America: Human Kinetics, 2004.

SOARES, D. C. et al. Ensino-aprendizagem e treinamento da finalização em jogos reduzidos no futebol. In:

ENCONTRO DE INICIAÇÃO CIENTÍFICA DA UNIPAR e FÓRUM DE PESQUISA DA UNIPAR, 14., 14., 2015, Umuarama. Anais... Umuarama: 2015.

TEOLDO, I. T. et al. Relação entre a dimensão do campo de jogo e os comportamentos táticos do jogador de futebol.

Revista Brasileira de Educação Física e Esporte, v. 25, n. 1, p. 79-96, 2011.

THOMAS, J. R.; NELSON, N.; SILVERMANN, S. J. Métodos de pesquisa em atividade física. 6. ed. Porto Alegre: Artmed, 2012.

VACARI, L. et al. Tomada de decisão tática dos jogadores de futebol nas categorias de base. In: ENCONTRO DE INICIAÇÃO CIENTÍFICA DA UNIPAR e FÓRUM DE PESQUISA DA UNIPAR, 14., 14., 2015, Umuarama. Anais... Umuarama: 2015.

ZISKIND. F. S. Jogos reduzidos e adaptados no futebol. Universidade do Futebol. Disponível em: $<$ http://www. universidadedofutebol.com.br>. Acesso em: 01 nov. 2011.

Recebido em: 05/05/2016 Aceito em: 01/11/2016 